\title{
Superconductor particles as the working media of a heat engine
}

\author{
P D Keefe \\ University of Detroit Mercy, Detroit, Michigan 48221 USA \\ E-mail: pdk@keefengine.com
}

\begin{abstract}
A heat engine is presented in which the working media comprises a multiplicity of mutually isolated particles of Type I superconductor which are selectively processed through H-T phase space so as to convert a heat influx from a high temperature heat reservoir into a useful work output, wherein little or no heat is rejected to a low temperature heat reservoir.
\end{abstract}

\section{Introduction}

Consider a heat engine which has a quantum working media in which, under predetermined conditions, a portion thereof undergoes a Bose-Einstein condensation, defined by a parameter, $\Delta_{T}$, the magnitude of which being temperature dependent, where $\Delta_{T}$ is maximum when the quantum working media is at absolute zero and vanishing when the quantum working media is at some finite temperature. The phase transition is first order, defined by the crossing of a boundary between two phases, one phase being designated as "normal" in which the quantum working media is everywhere uncondensed (that is, at a homogenous thermal state above the Fermi Sea), and the other phase being designated as "condensed" in which a first portion, 1- $\phi$, of the quantum working media is uncondensed and a second portion, $\phi$, of the quantum working media is quantum mechanically condensed (that is, in a quantum state below the surface of the Fermi Sea) by an amount related to $\Delta_{T}$.

An adiabatic phase transition of the quantum working media from the condensed phase to the normal phase will now be considered (a second adiabatic phase transition from the normal phase to the condensed phase is additionally involved in a full process cycle).

The phase transition proceeds as a mixing of two fluids, the normal portion, 1- $\phi$, with the condensed portion, $\phi$. Initially, the entropy will decrease by an amount,

$$
\Delta S_{1}=S_{A}-S_{B}=\int_{T_{A}}^{T_{B}}\left(C_{(1-\phi)} / T\right) d T
$$

due to the condensed portion, $\phi$, of the working media being quantum mechanically raised to the surface of the Fermi Sea, where $C_{(1-\phi)}$ is the specific heat of the normal portion of the quantum working media, $T_{A}$ is a starting temperature of the adiabatic phase transition process, and $T_{B}$ is an in-process intermediate temperature. Then, the entropy will increase by an amount,

$$
\Delta S_{2}=S_{C}-S_{B}=\int_{T_{0}}^{T_{C}} C_{\phi} d T-\int_{T_{B}}^{T_{C}}\left(C_{(1-\phi)} / T\right) d T
$$

due to the condensed portion of the working media being thermally raised from the surface of the Fermi Sea to a finite equilibrium entropy value of the entire quantum working media, where $\mathrm{C}_{\phi}$ is the specific heat of the condensed portion, $\mathrm{T}_{0}$ is absolute zero, and $\mathrm{T}_{\mathrm{C}}$ is an ending temperature of the adiabatic phase transition process.

It is an interesting that if $\Delta S_{1}>\Delta S_{2}$, as for example depicted by Figure 1, then a supra-Carnot efficiency of the phase transition process may be inferred. 


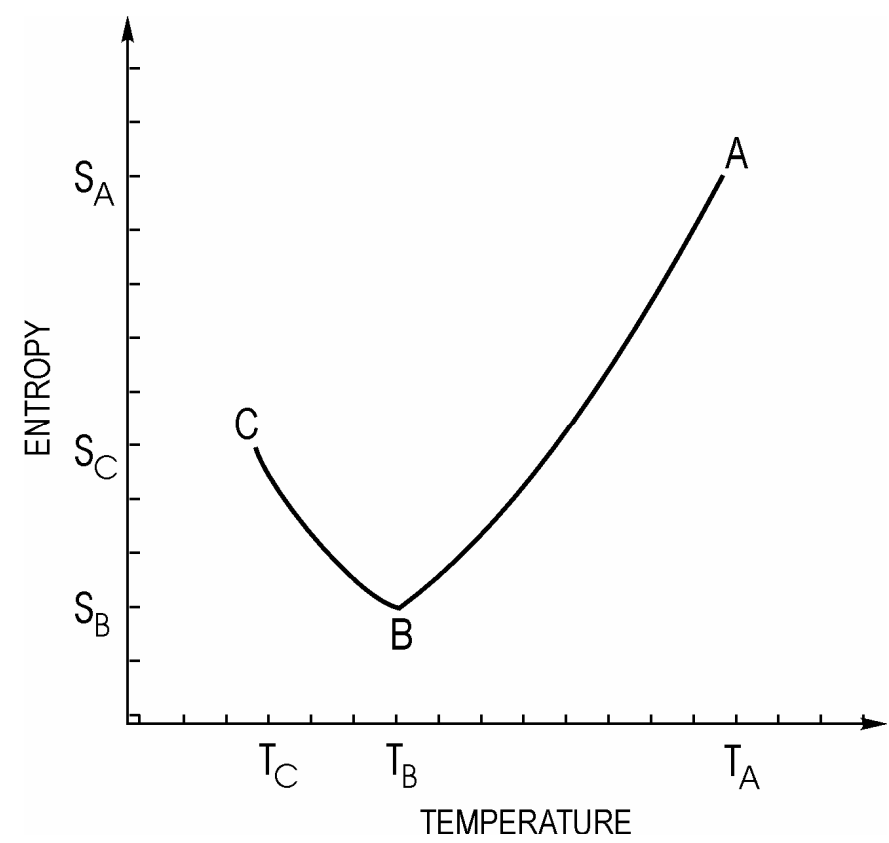

Fig. 1. A heat engine processes a quantum working media, wherein an adiabatic phase transition from the condensed to the normal phase involves a quantum mechanical reduction in entropy from point $\mathrm{A}$ to point $\mathrm{B}$, and thermal increase in entropy from point $\mathrm{B}$ to point $\mathrm{C}$, whereby the difference in entropy between points $\mathrm{A}$ and $\mathrm{C}$ involves a net lowering of the entropy of the quantum working media.

Three aspects of Type I superconductors suggest these are the ideal quantum working media for a heat engine, hereinafter referred to as a "superconductor heat engine." These aspects are: 1) the Meissner Effect, in which a magnetic field is ejected from a specimen interior by changing the temperature at constant magnetic field; 2) an appreciable range of coherence, $\xi(\mathrm{T})$, which is typically on the order of $10^{-4}$ $\mathrm{cm}$, whereby particles of this size may be considered as homogeneously coherent; and 3) the magnetocaloric effect, wherein the self-cooling/heating so provided yields a mechanism to process a superconductor through H-T space without thermal interaction with the external environment.

\section{Theory of a superconductor heat engine}

The concept of a heat engine utilizing a superconductor as the working media was first proposed by Chester [1] in 1962. Chester described an electrical generator embodiment in which a coil is wound around a superconductor core (i.e., the working media). Changes in the temperature in the presence of a fixed external magnetic field result in the superconductor undergoing a series of isothermal, first order phase transitions, wherein the magnetization change accompanying each phase transition results in an EMF in the coil that is available as a source of electrical energy. In this regard, Chester recognized that the Meissner effect is sine qua non to the process cycle in order to ensure an abrupt magnetic field expulsion at the phase transition of the working media from the normal phase to the superconductive phase. Chester reports that the efficiency of such a heat engine may be as high as $44 \%$ if niobium is chosen for the superconductor core.

In 1974, the author [2] proposed a superconductor heat engine in the form of a motor embodiment in which an armature composed of a superconductor working media moves in relation to a magnet. Figure 2 depicts a cycle of operation of the motor over four panels, wherein: the working media is in the normal phase while approaching a magnet (Panels 1 to 2); undergoes a phase transition from the normal phase to 
the superconductive phase accompanied by the Meissner effect (Panels 2 to 3); performs work as it recedes from the magnet (Panels 3 to 4); and undergoes a phase transition from the superconductive phase to the normal phase (Panels 4 to 1), whereupon the process cycle repeats.

\section{Panel 1}

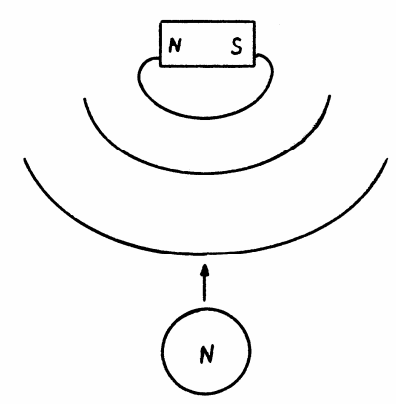

Panel 3

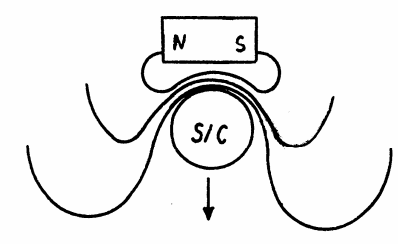

Panel 2

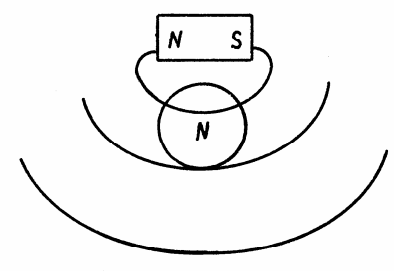

\section{Panel 4}

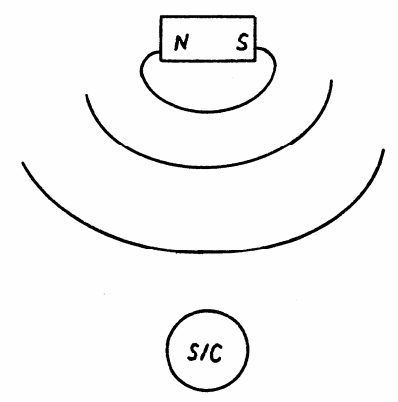

Fig. 2. Operational diagram of a superconductor heat engine in the form of a motor, where " $N$ " designates the normal phase, and " $S / C$ " designates the superconductive phase of the superconductor working media. The magnetic field distortion is emphasized at Panel 3 merely for illustrative purposes.

The $H-T$ phase space diagram for a conventional process cycle for a superconductor heat engine is depicted at Figure 3.

Beginning at point $\mathrm{A}$ in the superconductive phase at phase space coordinate $\left(H_{l}, T_{l}\right)$, the magnetic field is increased from $H_{1}$ to $H_{1}+\Delta H$, whereupon an isothermal, first order phase transition from the superconductive phase to the normal phase transpires. A latent heat of cooling evolves due to the difference in entropy between the superconductive and normal phases, and is provided by a high temperature heat reservoir. The latent heat may be calculated based upon the assumption that the Tuyn curve is parabolic [3], and is given by:

$$
L H_{1}=\left(H_{0}^{2} / 2 \pi T_{c}\right)\left(T_{1} / T_{c}-\left(T_{1} / T_{c}\right)^{3}\right)
$$

where $T_{c}$ is the critical temperature at zero magnetic field, and $H_{0}$ is the critical magnetic field at absolute zero. 


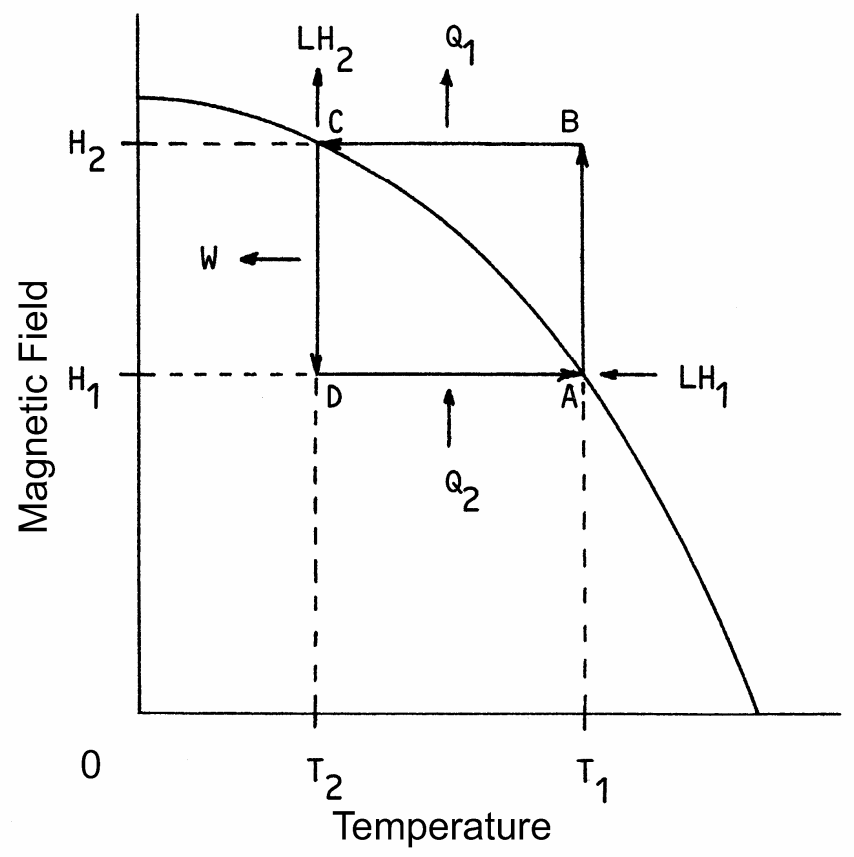

Fig. 3. $H-T$ space diagram for the conventional process cycle of a superconductor heat engine. A superconductor working media is processed between the normal and superconductive phases, each phase transition being isothermal and first order. The parabola is the Tuyn curve, which demarcates the phases.

The magnetic field is raised from Point $\mathrm{A}$ to Point $\mathrm{B}$, involving a free-space magnetic field work input. This free space magnetic field work is identically output going from Points $\mathrm{C}$ to $\mathrm{D}$, so this work function can be ignored.

Between Points B and C, the temperature of the superconductor working media is lowered by contact with a low temperature heat reservoir. The heat extracted is given by:

$$
Q_{1}=\int_{T_{1}}^{T_{2}} C_{n} d T
$$

where $C_{n}$ is the normal phase specific heat of the superconductor media.

At point $\mathrm{C}$ in the normal phase at phase space coordinate $\left(H_{2}, T_{2}\right)$, the magnetic field is decreased from $\mathrm{H}_{2}$ to $\mathrm{H}_{2}-\Delta H$, whereupon an isothermal, first order phase transition from the normal phase to the superconductive phase transpires, accompanied by the Meissner effect. A latent heat of heating is rejected to the low temperature heat reservoir, given by:

$$
L H_{2}=\left(H_{0}^{2} / 2 \pi T_{c}\right)\left(T_{2} / T_{c}-\left(T_{2} / T_{c}\right)^{3}\right) .
$$

The magnetic field is lowered from Point $\mathrm{C}$ to Point $\mathrm{D}$, involving a diamagnetic work output with respect to the superconductor working media due to the diamagnetism adduced by the Meissner effect (wherein the free-space magnetic field work output is ignored). This diamagnetic work output is given by:

$$
W=\int_{H_{2}}^{H_{1}} M d H
$$

where $\mathrm{M}$ is the magnetization of the superconductor working media.

Finally, the cycle is completed by a heat influx from the high temperature heat reservoir between Points $\mathrm{D}$ and $\mathrm{A}$, raising the temperature of the superconductor working media from $T_{2}$ to $T_{1}$ while in the 
superconductive phase. The heat influx is given by:

$$
Q_{2}=\int_{T_{2}}^{T_{1}} C_{s} d T .
$$

The efficiency of the conventional process cycle is given by:

$$
\eta=\left[1-\left(Q_{1}+L H_{2}\right) /\left(Q_{2}+L H_{1}\right)\right] x 100 .
$$

\section{Redisigning the conventional process cycle}

\section{The Magneto-Caloric Effect}

It would be very desirable if somehow the conventional superconductor heat engine process cycle of Figure 2 could be redesigned to improve the efficiency by eliminating the heat extraction process between Points B and C, and the heat rejection at Point $\mathrm{C}$.

It is well established that an adiabatic phase transition of a Type I superconductor at finite temperature involves the latent heat evolution being supplied (superconductive phase to normal phase) or absorbed (normal phase to superconductive phase) by the normal regime (the lattice and normal conduction electrons) of the superconductor [4].

Accordingly, if the isothermal phase transitions at Points $\mathrm{A}$ and $\mathrm{C}$ are replaced by adiabatic phase transitions, then, in theory, a magneto-caloric magnetization could cool the superconductor working media from Point $\mathrm{A}$ to Point $\mathrm{C}$, and a magneto-caloric demagnetization would then heat the superconductor from Point $\mathrm{C}$ to Point A without rejecting heat.

Problematically, such a process cycle modification, while solving the encumbrances of heat extraction and low temperature heat rejection, is unable to provide a useful heat engine, in that the process paths on either side of the Tuyn curve are identical, whereby there is no heat input and there is no net work output. The underlying reason for this is that the magneto-caloric process is isentropic, involving an intermediate state comprising domains of normal and superconductive phase, the relative proportion of which being a linear function of the instant strength of the applied magnetic field [5].

\section{Type I Superconductor Particles}

Pippard [6] first proposed in 1952 that a particle of Type I superconductor having a diameter not larger than the coherence length, $\xi(T)$, cannot experience an intermediate state due to the inability of the ordering parameter of the superelectrons to go to zero in a shorter distance. Experimental support for this hypothesis is reported by Lutes and Maxwell [7] who report that the phase transition of tin whiskers is abrupt in a transverse magnetic field, even though the demagnetizing factor is one-half.

An important consideration for particulate Type I superconductors is the affect size has on the energy gap. In this respect, for large size particles, the energy gap is unaffected and the phase transition at finite temperature is fully first order. However, for smaller size particles, the energy gap is diminished and tends toward a second order phase transition. Douglass [8] has considered the effect of thin film thickness on the energy gap, and reports that for Type I superconductor films having a thickness, $d$, larger than about five times the (magnetic field) penetration depth, $\lambda(T)$, the phase transition is substantially first order; however, diminution of the latent heat is expected for smaller diameters, disappearing in a second order phase transition for thicknesses smaller than $\sqrt{ } 5 \lambda(T)$.

Another important consideration with respect to particles of Type I superconductor is how the specific heat function is affected by size. Tsuboi and Suzuki [9] have considered the specific heat function for increasingly smaller tin particles, and report that for particles of a size equal to the range of coherence, the bulk specific heat function continues to pertain. 


\section{Conclusion}

A Type I superconductor particle having a diameter, $d$, defined by $5 \lambda(T)<d \leq \xi(T)$, hereinafter referred to as a "coherent particle" exhibits a phase transition that is fully first order, abrupt (i.e., without appearance of the intermediate state), and describable in terms of bulk thermodynamics. Examples of substances which can be fashioned into coherent particles are: aluminum (most preferred), indium and tin.

A coherent particle used as the working media of a superconductor heat engine has the potential to radically change [10] the conventional process cycle of Figure 3.

\section{Coherent magneto-caloric effect process cycle}

Figure 5 depicts a Coherent Magneto-Caloric Effect process cycle in $H-T$ phase space for a superconductor heat engine (see Fig. 2) in which the phase transitions are adiabatic and the superconductor working media is a coherent particle [11].

Beginning at point $\mathrm{A}$ in the superconductive phase at phase space coordinate $\left(H_{l}, T_{l}\right)$, the magnetic field is increased from $H_{1}$ to $H_{1}+\Delta H$ to Point $\mathrm{B}$, whereupon an adiabatic, first order phase transition from the superconductive phase to the normal phase transpires. A latent heat of cooling evolves, defined by (1), proceeding as a Coherent Magneto-Caloric Effect process, whereby the coherent particle cools from $T_{1}$ at Point $B$ to $T_{2}$ at Point $C$, defined by:

$$
L H_{1}=\int_{T_{1}}^{T_{2}} C_{n} d T .
$$

Simultaneously with the Coherent Magneto-Caloric Effect cooling, the magnetic field is raised from Point $\mathrm{B}$ to Point $\mathrm{C}$ involving a free-space magnetic field work input, which, as mentioned with respect to Figure 2, may be ignored.

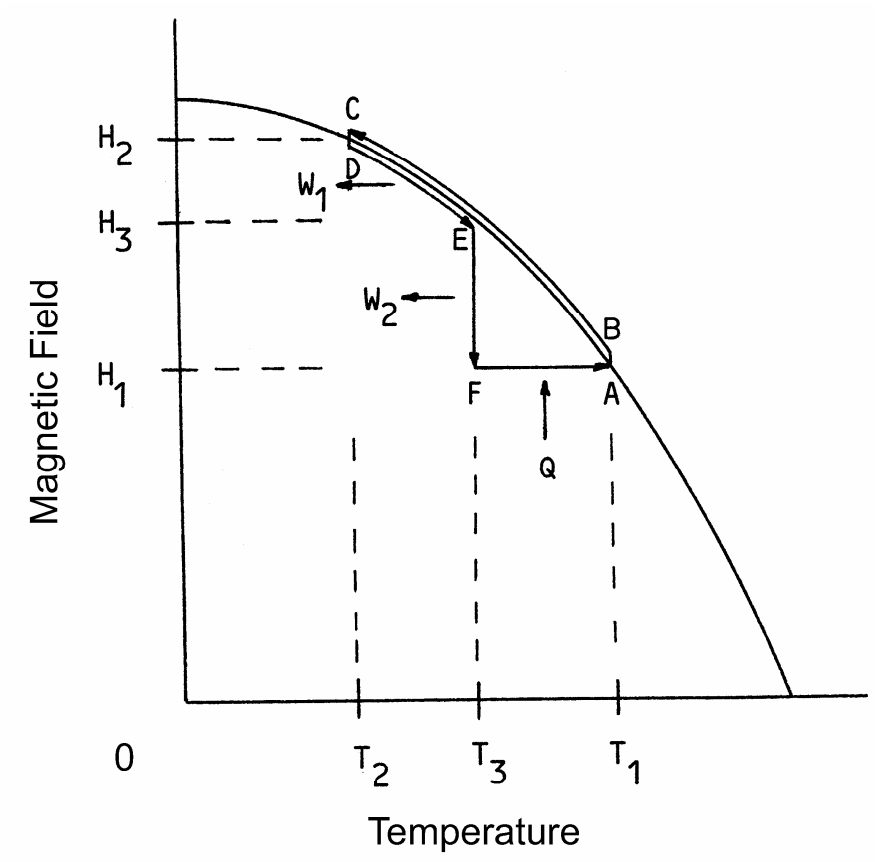

Fig. 5. H-T space diagram for a heat engine processing a "coherent particle" (a Type I superconductor having a diameter, $d$, defined by $5 \lambda(T)<d \leq \xi(T)$ ), between the normal and superconductive phases, each phase transition being 
adiabatic and first order. The parabola is the Tuyn curve, which demarcates the phases.

The magnetic field must be increased at a rate at least as fast as is necessary to keep the normal phase transition in process, given by:

$$
H_{i}>H_{0}\left(1-\left(T_{i}^{2} / T_{c}^{2}\right)\right)
$$

where $H_{i}$ is the instantaneous magnetic field, and $T_{i}$ is the instantaneous temperature of the coherent particle.

At point $\mathrm{C}$ in the normal phase at phase space coordinate $\left(H_{2}, T_{2}\right)$, the magnetic field is decreased from $\mathrm{H}_{2}$ to $\mathrm{H}_{2}-\Delta H$ to Point $\mathrm{D}$, whereupon an adiabatic, first order phase transition from the normal phase to the superconductive phase transpires, accompanied by the Meissner effect. A latent heat of heating evolves, defined by (3), proceeding as a Coherent Magneto-Caloric Effect process, whereby the coherent particle heats from $T_{2}$ at Point $\mathrm{D}$ to $T_{3}$ at Point $\mathrm{E}$, defined by:

$$
L H_{2}=\int_{T_{2}}^{T_{3}} C_{s} d T .
$$

Simultaneously with the Coherent Magneto-Caloric Effect heating, the magnetic field is decreased from Point D to Point E involving a diamagnetic work output with respect to the coherent particle due to the diamagnetism adduced by the Meissner effect (the free-space magnetic field work output being ignored). This diamagnetic work output is given by:

$$
W_{1}=\int_{H_{2}}^{H_{3}} M d H
$$

where $\mathrm{M}$ is the magnetization of the coherent particle.

The magnetic field must be decreased at a rate at least as fast as necessary to keep the superconductive phase transition in process, given by:

$$
H_{i}<H_{0}\left(1-\left(T_{i}^{2} / T_{c}^{2}\right)\right) \text {. }
$$

The magnetic field is then lowered further (at any convenient rate) from Point $\mathrm{E}$ to Point $\mathrm{F}$, involving an additional diamagnetic work output because of the continued diamagnetism of the coherent particle (the free-space magnetic work output also continuing to be ignored), given by:

$$
W_{2}=\int_{H_{3}}^{H_{1}} M d H .
$$

Finally, the process cycle is completed by a heat influx from a high temperature heat reservoir from $T_{3}$ to $T_{1}$ while in the superconductive phase. This heat influx is given by:

$$
Q=\int_{T_{3}}^{T_{1}} C_{s} d T .
$$

\section{Considerations}

\section{Possible Process Cycles}

The term "Coherent Magneto-Caloric Effect" process cycle is a generic term, in that the above described process cycle is, more particularly, a "Coherent Magneto-Caloric Effect Magnetization" process cycle, where the initial phase transition is from the superconductive phase to the normal phase. Other process cycles are possible [12]: a "Coherent Magneto-Caloric Demagnetization" process cycle, which involves an initial phase transition from the normal phase to the superconductive phase; and an "Enhanced Coherent Magneto-Caloric Demagnetization" process cycle, which further involves the heat 
influx being supplied at all times during the process cycle.

\section{Possible Embodiments}

The work output may be mechanical or electrical, depending upon the type of heat engine providing the Coherent Magneto-Caloric Effect process cycle [13]. In a first example, a heat engine in the form of a motor embodiment provides a mechanical work output, as for example shown at Figure 2. An armature is composed of a multiplicity of mutually isolated, coherent particles, and moves with respect to annularly arranged permanent magnets or electromagnets, wherein the armature is connected to a rotating shaft. In a second example, a heat engine in the form of a generator embodiment provides an electrical work output. A core is composed of a multiplicity of mutually isolated, coherent particles, and is exposed to time varying magnetic field intensity electromagnets, wherein a coil wound around the core provides a resulting EMF to an external circuit, as for example shown at Figure 6.
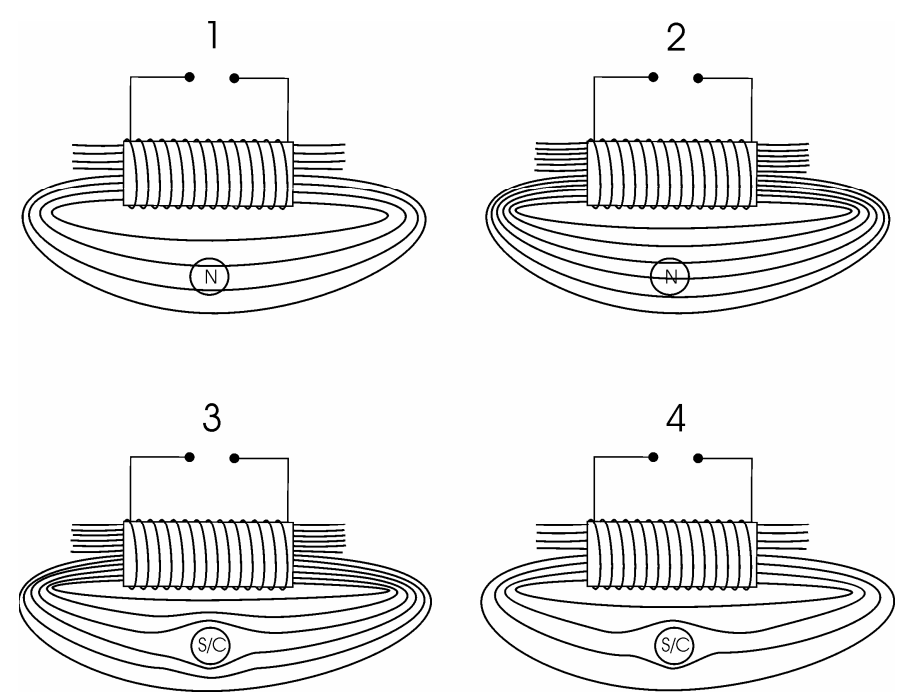

Fig. 6. Operational diagram of a superconductor heat engine in the form of a generator, where " $\mathrm{N}$ " designates the normal phase, and "S/C" designates the superconductive phase of the superconductor working media. An external circuit (not shown) connects to the electrodes of the excitation coil.

In Figure 6, a superconductor working media is in the normal phase while the magnetic field of an excitation coil is increasing (Panels 1 to 2); undergoes an isothermal phase transition from the normal phase to the superconductive phase accompanied by the Meissner effect when at the highest magnetic field value (Panels 2 to 3); performs work in the form of a net EMF work output in the excitation coil as the magnetic field is decreased (Panels 3 to 4); and undergoes an isothermal phase transition from the superconductive phase to the normal phase when at the lowest magnetic field value (Panels 4 to 1 ).

\section{Source of Possible Irreversible Losses}

Under ideal conditions, $W_{1}+W_{2}=Q$. Bardeen [15] has considered the Coherent MagnetoCaloric Effect process cycle, and proposed that at the adiabatic phase transitions, magnetic hysteresis would exhibit (ie., superheating at Point A and supercooling at Point $\mathrm{C}$ ), thereby rendering the process cycle subject to significant irreversible losses. However, Pippard [6] investigated the origin of phase transition magnetic hysteresis of superconductor particles, and predicted a result from which an inference 
may be drawn [16] that it is possible to minimize, or even eliminate, phase transition magnetic hysteresis.

\section{Ongoing Experiments}

The author is currently partnering [17] with A. V. Nikulov and S. V. Dubonos of the Institute for Microelectronics Technology, Russian Academy of Sciences, Chernolgolovka, Russia. The planned investigations on coherent particles of indium include observation of magnetic hysteresis effects during isothermal and adiabatic phase transitions. A ballistic Hall micromagnetometer [18] will be used to detect magnetization changes of individual coherent particles.

\section{REFERENCES}

[1] M. Chester, “Thermodynamics of a superconducting energy converter," J. Appl. Phy., Vol. 33, No. 2, pp. 643$647,1962$.

[2] P. D. Keefe, "A thermodynamic comparison between the magneto-mechanically and magneto-calorically induced superconductive phase transitions in a Type I superconductor culminating in a proposal for a new type of superconductive motor," Thesis, University of Detroit, Detroit, MI (1974).

[3] F. London, Superfluids, Vol. I, New York: Dover Publications, 1961, pg. 22.

[4] M. Yaqub, "Cooling by adiabatic magnetization of superconductors, Cryogenics, Vol. 1, Dec., pp. 101-107, 1960.

[5] R. L. Dolecek, “Adiabatic magnetization of a superconducting sphere,” Phys. Rev. Vol. 96, No. 1, pp. 25-28, 1954.

[6] A. B. Pippard, "On the magnetic hysteresis in superconducting colloids," Phil. Mag., Vol. 43, No. 338, pp. 273284, March, 1952.

[7] O. S. Lutes and E. Maxwell, "Superconducting transitions in tin whiskers," Phys. Rev., Vol. 97, pp. 1718-1720, 1955.

[8] D. H. Douglass, Jr., "Magnetic field dependence of the superconducting energy gap," Phys. Rev. Lett., Vol. 6, No. 7, pp. 346-348, 1961.

[9] T. Tsuboi and T. Suzuki, "Specific heat of superconducting fine particles of tin," J. Phys. Soc. of Jap., V. 42, No. 2, pp. 437-444, 1977.

[10] P. D. Keefe, "Second law implications of a magneto-caloric effect adiabatic phase transition of Type I superconductor particles," J. of Mod. Optics, Vol. 51, No. 16-18, pp. 2727-2730, 2004.

[11] P. D. Keefe "Coherent magneto-caloric effect heat engine process cycle," Quantum Limits to the Second Law: First international Conference, AIP Proceedings CP643, pp. 213-218, 2002.

[12] P. D. Keefe, "Coherent magneto-caloric effect superconductive heat engine process cycle," J. of Mod. Optics, Vol. 50, No. 15-17, pp. 2443-2454, 2003.

[13] P. D. Keefe, "Coherent magneto-caloric effect heat engine process cycle," U.S. Patent 4,638,194, 1987.

[14] P. D. Keefe, "Second law violation by magneto-caloric effect adiabatic phase transition of Type I superconductor particles," Entropy, www.mdpi.org/entropy, Vol. 6, pp. 116-127, 2004.

[15] J. Bardeen, Private letter communication to the author, Loomis Laboratory of Physics, Univ. of Ill., March 25, 1987.

[16] P. D. Keefe, "Quantum limit to the second law by magneto-caloric effect, adiabatic phase transition of mesoscopic-size Type I superconductor particles,” Physica E, Vol. 29, pp. 104-110, 2005.

[17] P. D. Keefe, author website, www.Keefengine.com, 2000-2007.

[18] A. K. Geim, S. V. Dubonos, J. G. S. Lok, I. V. Grigorieva, J. C. Maan, L. Theil Hansen and P. E. Lindelof, "Ballistic Hall micromagnetometry," J. Appl. Phy., Vol. 71, pp. 2379-2381, 1997. 